\title{
30 ANOS DE TEORIA DA GRAMÁTICA NA ANPOLL: UMA APRESENTAÇÃO
}

\section{YEARS OF GRAMMAR THEORY AT ANPOLL: AN OVERVIEW}

\author{
Danniel Carvalho \\ Universidade Federal da Bahia \\ Salvador, Bahia, Brasil \\ Lílian Teixeira de Sousa \\ Universidade Federal da Bahia \\ Salvador, Bahia, Brasil
}

RESUMO: Este texto traz um breve histórico do Grupo de Trabalho Teoria da Gramática (GTTG) da Associação Nacional de Pós-graduação e Pesquisa em Letras e Linguística (ANPOLL) desde sua fundação, em 1987, além de fazer uma apresentação do dossiê comemorativo de seus 30 anos.

PALAVRAS-CHAVE: Teoria da gramática; 30 anos; Anpoll.

ABSTRACT: This text shows a brief historical overview of the of the Grupo de Trabalho Teoria da Gramática (GTTG) of the Associação Nacional de Pós-graduação e Pesquisa em Letras e Linguística (ANPOLL) since its foundation, in 1987, besides presenting the dossier celebrating its 30 year anniversary.

KEYWORDS: Grammar theory; 30 years; Anpoll. 


\section{INTRODUÇÃO}

Criado em 1987, o Grupo de Trabalho Teoria da Gramática (GTTG) da Associação Nacional de Pós-graduação e Pesquisa em Letras e Linguística (ANPOLL), reúne, desde sua fundação, pesquisadores brasileiros que se debruçam sobre o estudo de gramática, em especial de seu modelo gerativista. $O$ GTTG funciona a partir dos critérios estabelecidos na Resolução nº 01/1996 da Associação Nacional de Pós-graduação e Pesquisa em Letras e Linguística que dispõe sobre a criação e o funcionamento dos grupos de trabalho da ANPOLL.

Segundo Moura e colegas (2010, p. 121), o GTTG tem pelo menos três objetivos principais: (I) promover estudos formais sobre gramática; (II) incentivar a troca de experiências entre os membros do GTTG; e (III) dar visibilidade à produção científica do grupo.

O GTTG agrupa pesquisadores das áreas da Fonologia, Morfologia, Sintaxe, Semântica e Aquisição da Linguagem, credenciados em Programa de Pós-graduação em todo o Brasil. Essa heterogeneidade de áreas no estudo da gramática tem possibilitado um debate interdisciplinar de temas diversos que constituem um modelo mais formal de análise linguística, gerativista ou não, além de facilitar a discussão de fenômenos de interface e àqueles relacionados ao desenvolvimento linguístico na aquisição da linguagem e na variação e mudança linguística.

Com base nesses fatos, O GTTG tem estabelecido o diálogo com outros Grupos de Trabalho da ANPOLL, tais como o GT (Grupo de Trabalho) Sociolinguística, o GT de Psicolinguística, o GT de Línguas Indígenas, o GT de Linguagem e Surdez, o GT de Linguagem e Cognição (cf. MOURA et al., 2010). Esses momentos inter-GTs possibilitam a cooperação interdisciplinar e a integração das diferentes áreas de pesquisa vinculadas à ANPOLL, o que resulta, muitas vezes, em trabalhos conjuntos entre esses pesquisadores, tais como publicação de artigos, coorientações de trabalhos acadêmicos, participações em bancas etc.

Desde 2004, o funcionamento do GTTG segue uma linha de organização temática, buscando priorizar a discussão de temas específicos, os quais podem ser tomados como diretivas de pesquisa para aqueles membros que queiram desenvolver um trabalho de cooperação entre as Universidades, com vistas à criação de intercâmbios e de formação de redes de pesquisa interinstitucionais. Esses temas, sugeridos pelos membros do Grupo de Trabalho, também orientam a organização dos Encontros anuais do GTTG, em que se tem procurado contar com a participação de pesquisadores das mais variadas Universidades do Brasil (na qualidade de apresentadores e debatedores), assim como com a participação de membros de outros GTs, como mencionado anteriormente.

$\mathrm{O}$ presente texto oferece, em um primeiro momento, um apanhado resultante de um levantamento de informações fundamentais da história do GTTG, colhidos de diversas fontes na internet, alguns podem ser verificados nas notas de rodapé no decorrer do texto, sendo a maioria informações dos relatórios de atividades do grupo e de depoimentos de seus membros fundadores, além de 
dois artigos que contam parte da história do grupo: Mioto (1994) e Moura et al. (2010), ambos publicados na Revista da ANPOLL. Os fatos aqui apresentados possibilitarão a manutenção de parte da memória do GT e servirão de fonte para contarmos um pouco da história da própria Teoria da Gramática no Brasil. Em um segundo momento, apresentamos o dossiê comemorativo dos 30 anos do GT Teoria da Gramática da ANPOLL, organizado por Danniel da Silva Carvalho e Lílian Teixeira de Sousa, atuais coordenador e vice-coordenadora do grupo.

\section{ESTRUTURA DO GTTG EM SEUS 30 ANOS}

Mioto (1994) informa que a ideia da criação de um Grupo de Trabalho Teoria da Gramática surgiu em um encontro sobre Gramática Gerativa realizado em Campinas em 1987 e idealizado por Charlotte Galves. A pesquisadora, então, realiza o primeiro encontro do GTTG em 1988, no Rio de Janeiro. Em 1989, Mary Kato realiza o segundo encontro do GTTG na PUC-São Paulo (Pontifícia Universidade Católica de São Paulo).

Charlotte Galves retorna à coordenação do GTTG no ano seguinte e realiza o terceiro encontro do GTTG em Recife, em 1990. A partir deste ano, o grupo decide por coordenações bianuais, tendo como coordenadora para o biênio 19901992 Miriam Lemle, que organiza o encontro nacional do GTTG em Porto Alegre em 1992. O biênio 1992-1994 é coordenado por Lúcia Lobato, que realiza o encontro nacional do GT em Caxambu em 1994. Neste ano, Carlos Mioto é eleito coordenador para o biênio 1995-1996, realizando o Encontro Nacional em João Pessoa em junho de 1996, no qual é definido que os encontros seriam anuais, sendo um intermediário (Encontro Nacional do GTTG) e outro no Encontro Nacional da ANPOLL. O biênio 1997-1998 passa a ter coordenadora e vicecoordenadora, Sonia Cyrino e Maria Cristina Figueiredo Silva, respectivamente. O primeiro encontro intermediário se deu no dia 9 de abril de 1997, na Universidade Federal de Santa Catarina, Florianópolis. Em seguida, o GTTG reuniu-se no XIII Encontro Nacional da ANPOLL, realizado de 10 a 12 de junho de 1998, na UNICAMP (Universidade Estadual de Campinas), Campinas. A gestão seguinte, de 1999-2000 teve como coordenadora e vice-coordenadora Esmeralda Negrão e Ilza Ribeiro. O encontro de 1999 ocorreu na USP (Universidade de São Paulo), São Paulo, e o encontro nacional, em 2000, ocorreu em Niterói, Rio de Janeiro.

Em 2002, o GT Teoria da Gramática da ANPOLL reuniu-se durante o XVI Encontro da ANPOLL, que foi organizado pela Instituto de Letras da Universidade Federal do Rio Grande do Sul em Gramado, RS, nos dias 5, 6 e 7 de julho. Nessa ocasião, o encontro do GT estava sob a coordenação de Maria Cristina Figueiredo Silva. Não houve encontro em 2001. A coordenação do GTTG ficou sob responsabilidade de Evani Viotti e Ana Paula Scher no biênio 2003-2004. O Encontro de 2003 ocorreu nos dias 4 e 5 de dezembro de 2003, na Faculdade de Filosofia, Letras e Ciências Humanas da USP, e o encontro de 2004 ocorreu nos dias 30 de junho a 2 de julho, em Maceió, no XIX Encontro Nacional 
da ANPOLL. Maria José Foltran e Márcia Cançado coordenaram o GTTG no biênio 2005-2006. O Encontro de 2005 ocorreu nos dias 1 e 2 de dezembro de 2005, em Ouro Preto MG, e o encontro de 2006 se deu nos dias 19, 20 e 21 de julho, durante o XXI Encontro Nacional da ANPOLL na PUC-São Paulo. O encontro de 2007 ocorreu nos dias 27 e 28 de setembro, na UNICAMP, Campinas, sob a coordenação de Ruth Lopes e Sonia Cyrino. O encontro de 2008 ocorreu durante o XXIII ENANPOLL (Encontro Nacional da ANPOLL), na Universidade Federal de Goiás, em Goiânia, de 2 a 4 de julho. A coordenação do biênio 2009-2010 foi representada pelas professoras Rozana Reigota Naves e Heloisa Maria M. L. Salles, respectivamente coordenadora e vice-coordenadora, com sede na Universidade de Brasília. O Encontro Nacional do GTTG em 2009 foi realizado na Universidade de Brasília, nos dias 26 e 27 de novembro, e o Encontro de 2010, inserido na programação geral do XXV ENANPOLL, foi realizado nos dias 02 e 03 de julho de 2010, na Universidade Federal de Minas Gerais (Belo Horizonte).

O biênio 2011-2012 foi coordenado por Maria Denilda Moura e Danniel da Silva Carvalho. O Encontro Nacional do GTTG foi realizado em Maceió, em setembro de 2011, e o encontro do GTTG no XXVII Encontro Nacional da ANPOLL, realizado no Instituto de Letras da UFF (Universidade Federal Fluminense), de 06 a 08 de julho de 2012. Marcello Modesto e Maria Aparecida Torres Morais foram eleitos coordenador e vice-coordenadora para a nova gestão do GTTG, no período 2013-2014. O Encontro Nacional foi realizado na USP, São Paulo, entre 5 e 9 de agosto de 2013, e o encontro do GTTG no XXIX Encontro Nacional da ANPOLL, nos dias 9, 10 e 11 de junho, Na Universidade Federal de Santa Catarina, em Florianópolis. O biênio 2015-2016 foi coordenado por Fábio Bonfim e Cilene Rodrigues. O Encontro Nacional do GTTG realizou-se nos dias 29 e 30 de julho de 2015, na Universidade Federal de Minas Gerais, em Belo Horizonte, e o do GTTG no XXXI Encontro Nacional da ANPOLL, na UNICAMP, Campinas.

O biênio 2017-2018 foi coordenado por Danniel da Silva Carvalho e Lílian Teixeira de Sousa. O Encontro Nacional de 2017 do GTTG foi comemorativo de seus 30 anos e foi realizado entre os dias 5 e 7 de julho de 2017 na Universidade Federal da Bahia. O Encontro Nacional de 2018 do GTTG aconteceu nos dias 28 e 29 de junho de 2018, na Universidade Federal de Santa Catarina, em Florianópolis.

Apresentamos, no Quadro 1, abaixo, um resumo das coordenações e encontros desses 30 anos do GT Teoria da Gramática. O GTTG, em sua história, realizou 27 encontros em 15 diferentes locais de realização, com 17 coordenações, 23 coordenadores e vice-coordenadores. 
Quadro 1: GTTG em números

\begin{tabular}{|c|c|c|c|c|}
\hline Encontro & Período & Coordenadores & Local & Membros $^{1}$ \\
\hline $1^{\mathrm{o}}$ & $1987-1988$ & $\begin{array}{c}\text { Charlotte Galves } \\
\text { (UNCAMP) }\end{array}$ & $\begin{array}{l}\text { UNICAMP, } \\
\text { Campinas-SP }\end{array}$ & - \\
\hline $2^{\circ}$ & 1989 & $\begin{array}{c}\text { Mary Kato } \\
\text { (UNICAMP) }\end{array}$ & $\begin{array}{l}\text { PUC-SP, São } \\
\text { Paulo-SP }\end{array}$ & - \\
\hline $3^{\circ}$ & 1990 & $\begin{array}{l}\text { Charlotte Galves } \\
\text { (UNCAMP) }\end{array}$ & $\begin{array}{c}\mathrm{UFPE}^{2}-\text { Recife- } \\
\text { PE }\end{array}$ & - \\
\hline $4^{\circ}$ & 1991-1992 & Miriam Lemle (UFRJ) & Porto Alegre-RS & - \\
\hline $5^{\circ}$ & 1993-1994 & Lúcia Lobato (UNB) & Caxanbu-MG & \\
\hline $6^{\mathrm{o}}$ & 1995-1996 & Carlos Mioto (UFSC) & $\begin{array}{c}\text { UFSC }^{3}, \\
\text { Florianópolis-SC }\end{array}$ & $\begin{array}{l}\text { Cerca de } \\
50^{4}\end{array}$ \\
\hline $7^{\circ}$ & \multirow[b]{2}{*}{ 1997-1998 } & \multirow{2}{*}{$\begin{array}{c}\text { Sônia Cyrino (UEL) e } \\
\text { Maria Cristina } \\
\text { Figueiredo Silva } \\
\text { (UFSC) }\end{array}$} & $\begin{array}{c}1997-\text { UFSC, } \\
\text { Florianópolis-SC }\end{array}$ & \multirow[b]{2}{*}{$72^{5}$} \\
\hline $8^{\circ}$ & & & $\begin{array}{c}1998- \\
\text { UNICAMP, } \\
\text { Campinas-SP }\end{array}$ & \\
\hline $9^{\circ}$ & \multirow{2}{*}{$1999-2000$} & \multirow{2}{*}{$\begin{array}{l}\text { Esmeralda Negrão } \\
\text { (USP) e Ilza Ribeiro } \\
\text { (UNIFACS) }\end{array}$} & $\begin{array}{c}1999 \text { - USP, São } \\
\text { Paulo-SP }\end{array}$ & \multirow[b]{2}{*}{-} \\
\hline $10^{\circ}$ & & & $\begin{array}{c}2000-\mathrm{UFF}, \\
\text { Niterói-RJ }\end{array}$ & \\
\hline & \multirow{2}{*}{ 2001-2002 } & \multirow{2}{*}{$\begin{array}{c}\text { Maria Cristina } \\
\text { Figueiredo Silva } \\
\text { (UFSC) }^{6}\end{array}$} & $\begin{array}{c}2001 \text { - Não } \\
\text { houve encontro }\end{array}$ & \multirow[b]{2}{*}{ - } \\
\hline $11^{\circ}$ & & & $\begin{array}{c}2002-\text { UFRS, } \\
\text { Gramado-RS }\end{array}$ & \\
\hline $12^{\circ}$ & \multirow{2}{*}{ 2003-2004 } & \multirow{2}{*}{$\begin{array}{c}\text { Evani Viotti (USP) e } \\
\text { Ana Paula Scher } \\
\text { (USP) }\end{array}$} & $\begin{array}{l}2003 \text { - USP, São } \\
\text { Paulo-SP }\end{array}$ & \multirow[b]{2}{*}{-} \\
\hline $13^{\circ}$ & & & $\begin{array}{c}2004-\mathrm{UFAL}^{7} \\
\text { Maceió-AL }\end{array}$ & \\
\hline $14^{\mathrm{o}}$ & 2005-2006 & $\begin{array}{c}\text { Márcia Cançado } \\
\text { (UFMG) e Maria José }\end{array}$ & $\begin{array}{c}2005 \text { - Ouro } \\
\text { Preto-MG }\end{array}$ & - \\
\hline
\end{tabular}

\footnotetext{
${ }^{1}$ Infelizmente, o número de membros filiados ao longo dos anos não pôde ser recuperado completamente.

${ }^{2}$ Universidade Federal de Pernambuco.

${ }^{3}$ Universidade Federal de Santan Catarina.

${ }^{4}$ Dados extraídos de Mioto (1994).

${ }^{5}$ Informações disponíveis em: $<$ http://www.unicamp.br/ anpoll/gt22.htm>. Acesso em 02/07/2017.

${ }^{6}$ Não há informações disponíveis sobre a vice-coordenação desse biênio.

${ }^{7}$ Universidade Federal de Alagoas.
} 


\begin{tabular}{|c|c|c|c|c|}
\hline Encontro & Período & Coordenadores & Local & Membros $^{1}$ \\
\hline $15^{\circ}$ & & Foltran (UFPR) & $\begin{array}{c}2006 \text { - PUC-SP } \\
\text { São Paulo-SP }\end{array}$ & \\
\hline $16^{\circ}$ & \multirow{2}{*}{ 2007-2008 } & \multirow{2}{*}{$\begin{array}{c}\text { Ruth Lopes } \\
\text { (UNICAMP) e Sonia } \\
\text { Cyrino (UNICAMP) }\end{array}$} & $\begin{array}{c}2007- \\
\text { UNICAMP, } \\
\text { Campinas-SP }\end{array}$ & \multirow[t]{2}{*}{$29^{8}$} \\
\hline $17^{\circ}$ & & & $\begin{array}{c}2008-\mathrm{UFG}^{9}, \\
\text { Goiânia-GO }\end{array}$ & \\
\hline $18^{\circ}$ & \multirow[b]{2}{*}{ 2009-2010 } & \multirow{2}{*}{$\begin{array}{c}\text { Rozana Reigota Naves } \\
\text { (UnB) e Heloisa Maria } \\
\text { M. L. Salles (UnB) }\end{array}$} & $\begin{array}{c}2009-\mathrm{UNB}^{10} \\
\text { Brasília-DF }\end{array}$ & \multirow[b]{2}{*}{$35^{11}$} \\
\hline $19^{\circ}$ & & & $\begin{array}{c}2010-\mathrm{UFMG}^{12} \text {, } \\
\text { Belo Horizonte- } \\
\text { MG }\end{array}$ & \\
\hline $20^{\circ}$ & \multirow{2}{*}{ 2011-1012 } & \multirow{2}{*}{$\begin{array}{c}\text { Maria Denilda Moura } \\
\text { (UFAL) e Danniel da } \\
\text { Silva Carvalho } \\
\text { (UFBA) }\end{array}$} & $\begin{array}{l}2011 \text { - UFAL, } \\
\text { Maceió-AL }\end{array}$ & \multirow{2}{*}{$43^{13}$} \\
\hline $21^{\circ}$ & & & $\begin{array}{c}2012 \text { - UFF, } \\
\text { Niterói-RJ }\end{array}$ & \\
\hline $22^{\circ}$ & \multirow{2}{*}{ 2013-2014 } & \multirow{2}{*}{$\begin{array}{l}\text { Marcello Modesto } \\
\text { (USP) e Maria } \\
\text { Aparecida Torres } \\
\text { Morais (USP) }\end{array}$} & $\begin{array}{c}2013 \text { - USP, São } \\
\text { Paulo-SP }\end{array}$ & \\
\hline $23^{\circ}$ & & & $\begin{array}{c}2014 \text { - UFSC, } \\
\text { Florianópolis-SC }\end{array}$ & \\
\hline $24^{\circ}$ & \multirow{2}{*}{ 2015-2016 } & \multirow{2}{*}{$\begin{array}{l}\text { Fábio Bonfim Duarte } \\
\text { (UFMG) e Cilene } \\
\text { Rodrigues (PUC-RJ) }\end{array}$} & $\begin{array}{c}2015 \text { - UFMG, } \\
\text { Belo Horizonte- } \\
\text { MG }\end{array}$ & \multirow{2}{*}{$61^{14}$} \\
\hline $25^{\circ}$ & & & $\begin{array}{c}2016- \\
\text { UNICAMP, } \\
\text { Campinas-SP }\end{array}$ & \\
\hline
\end{tabular}

\footnotetext{
${ }^{8}$ Informações disponíveis em: <http://anpoll.org.br/gt/teoria-da-gramatica-gttg/wpcontent/uploads/sites/14/2013/03/GTTeoriasdaGramaticaRelatorio2006-2008.pdf > .

Acesso em 02/07/2017.

${ }^{9}$ Universidade Federal de Goiás

${ }^{10}$ Universidade de Brasília.

${ }^{11}$ Informações disponíveis em: http://anpoll.org.br/gt/teoria-da-gramatica-gttg/wpcontent/uploads/sites/14/2013/03/relatorio-gttg-2008-2010.pdf. Acesso em 02/07/2017.

${ }^{12}$ Universidade Federal de Minas Gerais.

${ }^{13}$ Informações disponíveis em: http://anpoll.org.br/gt/teoria-da-gramaticagttg/relatorios-de-atividade/. Acesso em 02/07/2017.

${ }^{14}$ Informações disponíveis em: http://anpoll.org.br/gt/teoria-da-gramaticagttg/membros/. Acesso em 02/07/2017.
} 


\begin{tabular}{|c|c|c|c|c|}
\hline Encontro & Período & Coordenadores & Local & Membros ${ }^{1}$ \\
\hline $26^{\circ}$ & \multirow{2}{*}{$2017-2018$} & \multirow{2}{*}{$\begin{array}{l}\text { Danniel da Silva } \\
\text { Carvalho (UFBA) e } \\
\text { Lílian Teixeira de } \\
\text { Sousa (UFBA) }\end{array}$} & $\begin{array}{c}2017 \text { - UFBA, } \\
\text { Salvador-BA }\end{array}$ & 61 \\
\hline $27^{\circ}$ & & & $\begin{array}{l}2018-\text { UFSC, } \\
\text { Florianópolis-SC }\end{array}$ & $54^{15}$ \\
\hline
\end{tabular}

Fonte: os autores

\section{ESTRUTURA DO DOSSIEE COMEMORATIVO DOS 30 ANOS DO GRUPO DE TRABALHO TEORIA DA GRAMÁTICA (GTTG)}

O dossiê comemorativo dos 30 anos do Grupo de Trabalho Teoria da Gramática (GTTG) é o resultado da seleção dos trabalhos apresentados no Encontro Nacional do GTTG na Universidade Federal da Bahia, Salvador, entre os dias 5 e 7 de julho de 2017. O encontro contou com a participação das três primeiras coordenadoras do GTTG, Charlotte Galves (UNICAMP), Mary Kato (UNICAMP) e Maria Cristina Figueiredo Silva (UFPR), em uma mesa de abertura, com o tema "A categoria 'pronome' na Gramática Gerativa" e com uma conferência de Halldor Armann Sigurđsson (Lund University), com o título On Minimal Features. O evento contou ainda com duas sessões de debate com professores pesquisadores convidados: Filomena Sandalo (UNICAMP), Fábio Duarte (UFMG) e Maria Luísa Freitas (UFPE), discutindo fenômenos relacionados às línguas indígenas; e Sérgio Menuzzi (UFRGS - Universidade Federal do Rio Grande do Sul), Roberta Pires de Oliveira (UFSC) e Rerisson Cavalcante de Araújo (UFBA - Universidade Federal da Bahia), em um debate sobre semântica das línguas naturais. Marcaram presença ainda vários membros do GTTG e estudantes de diversos Programas de Pós-Graduação de várias regiões do Brasil, que apresentaram trabalhos em sessões de comunicação oral e de pôster.

O encontro possibilitou momentos de vigoroso debate científico de alta qualidade, dos quais boa parte tivemos a felicidade de poder registrar neste dossiê. $\mathrm{O}$ volume reúne trabalhos que discutem os mais diversos temas relativos à gramática das línguas naturais, contemplando áreas como Fonologia, Morfossintaxe, Semântica, abordando fenômenos de diferentes línguas, como o português, o inglês e línguas indígenas, entre outras.

O primeiro trabalho, de Bruna Karla Pereira, com o título $N U M P E$ SILENT NOUNS: FRONTEIRAS SINTÁTICAS NA MARCAÇÃO DE PLURAL $N O P B$, investiga a distribuição do morfema de plural, na estrutura interna do DP (Determiner Phrase), em português brasileiro não padrão (PB). A autora

\footnotetext{
${ }^{15}$ Informações disponíveis em: http://anpoll.org.br/gt/teoria-da-gramaticagttg/membros/. Acesso em 02/07/2017.
} 
argumenta que essa distribuição é determinada pela posição de cardinais ou de silent nouns, propondo que: (I) a posição dos cardinais divide o DP em dois domínios nos quais sintagmas à esquerda de NumP são marcados com o morfema '-s' de plural, enquanto sintagmas à direita de NumP não são; (II) a posição de silent nouns exerce basicamente essa mesma função, em estruturas nas quais os cardinais não podem aparecer; e (III) os traços de número são valorados e interpretáveis em Num e se tornam valorados em D (determiner) e $\mathrm{N}$ (noun) após checagem via concordância.

Em seguida, Carlos Felipe Pinto, com o trabalho A VARIAÇÃO QUE $x$ QUEM EM DUAS CONSTRUÇÕES DE CLIVAGEM, discute duas construções de clivagem no português para mostrar que, nessas construções não há uma oração relativa envolvida e que, em ambos os casos, a estrutura é a mesma, contendo uma oração completiva. $\mathrm{O}$ autor apresenta uma análise em que ambos os tipos de construções de clivagem são derivados através da mesma estrutura. É proposta uma análise unificada para as estruturas clivadas e pseudo-clivadas extrapostas tentando explicar a variação entre as duas construções. $\mathrm{O}$ autor, ainda, tece problemas residuais das questões levantadas neste trabalho que deverão ser debatidas em trabalhos futuros.

Eloísa Pilati, Rozana Naves e Heloisa Salles apresentam o artigo intitulado UMA ANÁLISE UNIFICADA PARA SUJEITOS INOVADORES (NULOS E MANIFESTOS) NA GRAMÁTICA DO PORTUGUÊS BRASILEIRO (PB), que teve como objetivos principais descrever e analisar o licenciamento de sujeitos inovadores (nulos e manifestos) no PB e apresentar dados da diacronia do sujeito no PB, em comparação com o português europeu, como evidência para a cisão no paradigma pronominal/flexional do $\mathrm{PB}$ e para a análise da ordem VS como inversão locativa nessa língua. Nesta abordagem, a categoria pronominal/flexional do PB na terceira pessoa é realizada como um feixe de traços phi, sendo a realização de um DP locativo (lexical ou nulo) na posição de sujeito determinada pela ausência do traço de referencialidade na terceira pessoa.

A seguir, Fernanda de Oliveira Cerqueira e Danniel da Silva Carvalho assinam o artigo SOBRE A DETERMINAÇÃO PRONOMINAL: O CASO DA REFERENCIALIDADE DO PRONOME DE TERCEIRA PESSOA NO PORTUGUESS BRASILEIRO, que teve por objetivo observar aspectos do comportamento morfossintático e semântico da forma pronominal plena de terceira pessoa no Português Brasileiro, a partir do inventário de traços que a constitui, sob a perspectiva de uma teoria de traços. Para tanto, foram observados dados do português brasileiro, os quais permitiram observar que o pronome pleno de terceira pessoa pode ocorrer em qualquer contexto sintático que admite apenas leitura definida e específica, exceto em posição de sujeito, que pode assumir leitura definida e/ou específica, ou ainda, nenhuma das duas (caso dos expletivos), e, portanto, terceira pessoa não apresenta comportamento arbitrário, como ocorre com a primeira e a segunda pessoa. 
Filomena Sandalo, com o trabalho EMPOBRECIMENTO E FISSÃO EM ASSIMETRIAS DE PESSOA NAS LÍNGUAS GUAYKURÚ, argumenta que alguns fatos de assimetrias de pessoa devam ser abordados como fenômenos de interface, ao invés operações puramente sintáticas. Argumenta neste trabalho que uma operação sintática desloca um argumento interno para o domínio de TP (Tense Phrase), voz inversa, e regras de reajuste pós-sintáticas derivam diferentes hierarquias de pessoa na morfologia de concordância.

Márcia Cançado, Luana Lopes Amaral e Letícia Lucinda Meirelles apresentam o trabalho VERBOWEB: UMA PROPOSTA DE CLASSIFICAÇÃO VERBAL. O artigo introduz o VerboWeb, um banco de dados lexicais do português brasileiro, em desenvolvimento, que conta atualmente com mais de 1360 verbos classificados em classes e em subclasses, de acordo com suas propriedades sintático-semânticas. As autoras apresentam uma proposta de classificação verbal por propriedades léxico-gramaticais, mostrando como se deu a classificação verbal utilizada no VerboWeb e os possíveis níveis de classificação dos verbos.

Em seguida, Mary Kato defende, em seu trabalho intitulado ASPECTOS MORFOFONOLÓGICOS NOS PARADIGMAS DOS PRONOMES FORTES E FRACOS, que os traços phi podem vir realizados nos pronomes fortes e nos tipos de pronomes fracos (pronomes livres, clíticos e flexão de concordância). Este trabalho propõe que a possibilidade de homofonia (rima) entre eles pode levar à elipse dos pronomes livres fracos, gerando o fenômeno do chamado sujeito nulo pro, e que é na morfofonologia que a criança se pauta para determinar o tipo de língua que ela está aprendendo. A autora assume esta proposta como base para a análise do Português Brasileiro com respeito ao Parâmetro do Sujeito Nulo.

Pablo Ribeiro, no artigo O PAPEL DAS NOÇÕES DE DINAMISMO, CAUSAÇÃO INTERNA E CONTROLE NA REPRESENTAÇÃO DOS VERBOS DINAMMICOS MONOARGUMENTAIS, discute o papel das noções de dinamismo, causação interna e controle na representação léxico-conceitual de diferentes classes de verbos dinâmicos monoargumentais no PB. Os objetivos principais do artigo são (I) analisar como estas propriedades influenciam o comportamento gramatical desses verbos e (II) definir qual a melhor maneira de representá-las na estrutura conceitual desses predicados, sob a luz da Semântica Conceitual. Para tanto, são examinados, inicialmente, alguns problemas da proposta de Jackendoff (1990) para o tratamento dos verbos de modo de movimento no inglês. Em seguida, são discutidas algumas análises clássicas das noções de controle, causação interna e eventos acionais na semântica lexical, que servirão como base para a análise apresentada neste trabalho. Por fim, é apresentada uma proposta de representação dos verbos dinâmicos monoargumentais que envolve os predicados primitivos MOVE (movimento) e ACT (ação), bem como os traços de controle e causação interna.

Raisa Reis dos Santos e Maria Cristina Vieira de Figueiredo Silva, em seu artigo OS TRAÇOS DE ASPECTO LEXICAL E AS NOMINALIZAÇÕES EM - 
ÇÃO NO PORTUGUÊS BRASILEIRO, discutem as nominalizações em -ção, com o objetivo de investigar os fatores que contribuem para que essas construções, no português brasileiro, denotem as diversas leituras observadas, evento, resultado e entidade. As autoras assumem que essas leituras estão relacionadas: (I) às fases por que passam essas formações durante a sua derivação, considerando os pressupostos teóricos da Morfologia Distribuída; e (II) à presença/ausência dos traços de aspecto lexical, dinamicidade, duração e telicidade nas nominalizações. É proposto que as formações com leituras de evento e resultado passam por uma projeção verbal e, consequentemente, por uma projeção aspectual e as nominalizações com leitura de entidade não passam por uma projeção verbal, dado que são formadas diretamente da raiz.

O penúltimo artigo deste dossiê é SOBRE A PRESSUPOSIÇÃO DAS CLIVADAS, de Sérgio Menuzzi, que apresenta um paradoxo relativo às propriedades pressuposicionais das clivadas e propõe uma solução para ele. A explicação proposta pelo autor para esse paradoxo é que uma asserção de identidade fornece uma resposta completa apenas quando há uma pressuposição contextual de unicidade - que não é propriedade inerente das perguntas $W H$.

Fechamos o dossiê com o trabalho de Sonia Maria Lazzarini Cyrino, intitulado ANIMACIDADE NA SINTAXE: UMA ABORDAGEM FORMAL PARA AS HIERARQUIAS DE REFERENCIALIDADE. O artigo investiga fenômenos que envolvem o traço animacidade, e que foram observados separadamente na literatura sobre as línguas românicas: Marcação Diferencial do Objeto, leísmo e a Restrição Caso-Pessoa. O objetivo foi analisar esses fenômenos em comparação com o objeto nulo do português brasileiro, com a finalidade de determinar o papel que a animacidade tem na sintaxe. Os resultados da pesquisa demonstram que, de fato, a animacidade é relevante para a sintaxe. A partir desses resultados, a autora avança em uma proposta que pretende dar conta dos fenômenos investigados e dá suporte a uma abordagem.

Para finalizar esta breve apresentação do dossiê comemorativo dos 30 anos do Grupo de Trabalho Teoria da Gramática (GTTG) da Associação Nacional de Pós-graduação e Pesquisa em Letras e Linguística (ANPOLL), gostaríamos de firmar alguns agradecimentos. Primeiramente, gostaríamos de agradecer a todos os autores que colaboraram com este volume pela confiança depositada em nós para sua organização. Em especial, aos colegas que aceitaram fazer parte de nosso encontro comemorativo de 30 anos do GTTG, mesmo sabendo da escassez de recursos com que contávamos para sua realização, nosso muitíssimo obrigado! Agradecemos também às editoras da Revista da Anpoll Andréia Guerini e Mailce Borges Mota por aceitarem a proposta do dossiê. O agradecimento também se estende ao Programa de Pós-Graduação em Língua e Cultura da Universidade Federal da Bahia (PPGLinC) e Coordenação de Aperfeiçoamento de Pessoal de Nível Superior (CAPES) pelo apoio na organização do evento. Esperamos que os trabalhos aqui reunidos sirva de inspiração para a continuação das pesquisas em teoria da gramática e, assim, continuar a alavancar o debate científico na área. 


\section{REFERÊNCIAS}

MIOTO, C. O GT de Teoria da Gramática. Anpoll, v. 1, n. 1, p. 9-11, 1994.

MOURA, M. D.; NAVES, R. R.; SALLES, H. M. M. L.; LOPES, R. E. V.; CANÇADO, M.; FOLTRAN, M. J.; SILVA, M. C. F.; FONSECA, H. D. C. Teoria da Gramática: tendências e perspectivas. Anpoll, v. 1, n. 29, p. 119-168, 2010.

Danniel Carvalho DannielCarvalho@ufba.br

Lílian Teixeira de Sousa LilianTSousa@gmail.com

Recebido em: 15 jan. 2018 Aceito em: 15 abr. 2018

Publicado em: 1 out. 2018 